\title{
Vision-based Force Feedback Estimation for Robot-assisted Surgery using Instrument-constrained Biomechanical 3D Maps
}

\author{
Nazim Haouchine ${ }^{1}$, Winnie Kuang ${ }^{2}$, Stephane Cotin ${ }^{1}$, and Michael Yip ${ }^{2}$
}

\begin{abstract}
We present a method for estimating visual and haptic force feedback on robotic surgical systems that currently do not include significant force feedback for the operator. Our approach permits to compute contact forces between instruments and tissues without additional sensors, relying only on endoscopic images acquired by a stereoscopic camera. Using an underlying biomechanical model built on-the-fly from the organ shape and by considering the surgical tool as boundary conditions acting on the surface of the model, contact force can be estimated at the tip of the tool. At the same time these constraints generate stresses that permit to compose a new endoscopic image as visual feedback for the surgeon. The results are demonstrated on invivo sequences of a human liver during robotic surgery, while quantitative validation is performed on an DejaVu and ex-vivo experimentation with ground truth to show the advantage of our approach.
\end{abstract}

Index Terms-Computer-aided Surgery, Force Feedback, Physics-based Simulation, Vision-based 3D Reconstruction.

\section{INTRODUCTION}

$\mathbf{R}$ OBOTIC-assisted minimally invasive surgery (RMIS) techniques have been developed to positively impact the patients' post-operative and intra-operative outcome. In such procedures, organs are manipulated indirectly via robotic instruments inserted through trocars while watching a monitor that retrieves the view captured via an endoscopic camera. This procedure reduces the risks of bleeding and hemorrhaging and shortens the recovery time which makes it a standard and wellestablished procedure.

The two main disadvantages of this type of procedures are: (1) a limited visual perception of the scene retrieved from the endoscopic camera and (2) the lack of interaction feedback since surgeons are not in direct contact with the organs, but with robotic interfaces (teleoperated from master console) that guide the instrument. This loss of feedback makes robotic procedures challenging and require additional decision support, especially for unexperienced surgeons.

Some solutions have been proposed to overcome the limited visual perception, mainly based on Augmented Reality techniques [1], [2], [3]. Force and visual feedback are negligible

Manuscript received: September 9, 2017; Revised December 22, 2017; Accepted February, 20, 2018

This paper was recommended for publication by Editor Ken Masamune upon evaluation of the Associate Editor and Reviewers' comments.

${ }^{1}$ Nazim Haouchine and Stephane Cotin are with the Mimesis Group, Inria, Strasbourg, France stephane.cotineinria.fr

${ }^{2}$ Winnie Kuang and Michael Yip are with the Advanced Robotics and Controls Lab, University of California, San Diego, CA, USA yip@ucsd.edu

Digital Object Identifier (DOI): see top of this page. in current surgical robotic systems despite their importance in improving surgical skills [4], [5]. This could be explained by the cost of implementing such sensors, in addition to the complex design requirements that the surgical environment imposes.

This work presents a novel method that permits to estimate, without additional sensors, the force between instruments and tissues when performing RMIS.

Our method uses a biomechanical map built on-the-fly from stereoscopic images using 3D reconstruction and meshing techniques. By populating the 3D map with mechanical tissue properties and tracking instrument/tissue non-rigid interaction, forces can be computed at the tip of the tool and can be transmitted to the surgeon as both visual and haptic feedback.

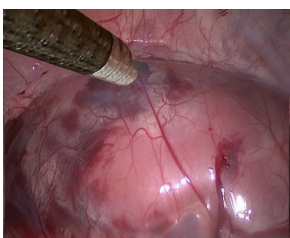

(a)

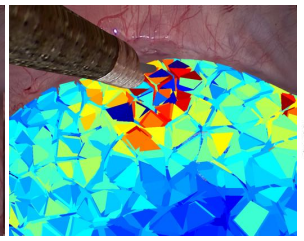

(b)

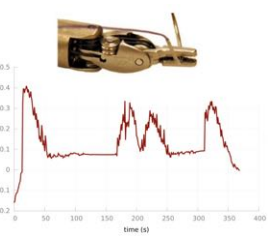

(c)
Fig. 1: Our method allows to estimate, using only the endoscopic images (a), the force feedback due to instrument/tissue contact for robot-assisted minimally invasive surgery, and compute both visual feedback (b) and force magnitude (c).

\section{RELATED WORKS}

Several methods for force feedback have been proposed in the literature relying on force sensors or vision-based sensors [6], [7].

The considerable amount of work relying on force sensors [8], [9], [10] has mainly focused on the development of miniaturized sensors to ease their integration with actual systems. In addition to their small size, they have to be water resistant and insensitive to changes in temperature and sterilizable. Although these methods are generally recognized to be efficient and accurate, their high cost makes them seldom used in current surgical robots.

Vision-based methods have been proposed as an alternative to force sensor approaches. They have the advantage of exploiting the already exiting endoscopic camera.

The method proposed by Gras et al. uses an inverse rendering method [11] to augment the surgical scene with 


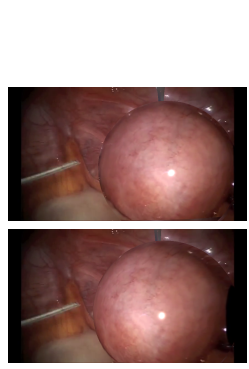

(a) input images

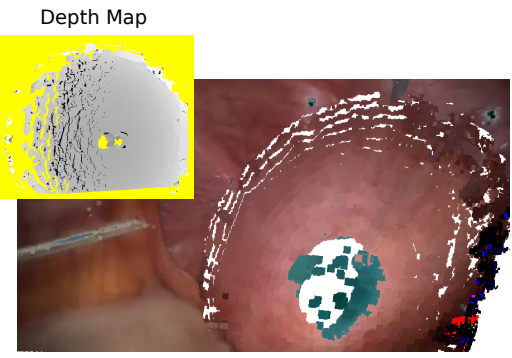

(b) 3D recontruction

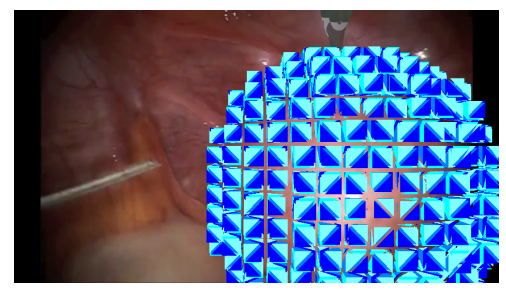

(c) 3D biomechanical map

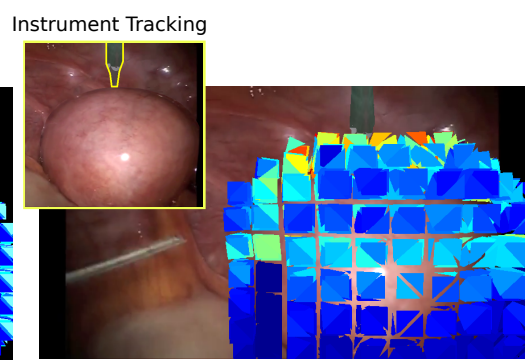

(d) Instrument/Tissue contact

Fig. 2: Method overview: our pipeline takes as an input the stereo endoscopic images (a) to perform a 3D reconstruction of the scene (b). From the 3D reconstruction, a biomechanical map is built using a smoothing algorithm followed by Delaunay triangulation (c). The instrument/tissue contact generates stresses on the biomechanical map that will overlay the visual force and measure force feedback (d).

visual force feedback. This method, dedicated to neurovascular surgery, detects the proximity of the instrument with the tissue and highlights contact area. The visualization is done in depth thanks to the use of a finite element model that interpolates veins and tumors. High-fidelity simulation has been considered in [12]. Based on volume rendering, a virtual environment mimicking the actual operation is built using pre-operative data. In [13], a hybrid method for haptic and visual feedback is proposed using a vision-based 3D map reconstruction, path tracking and movement control module to measure the distance between the surgeon's inputs and the map. In [14], the authors presented a dynamic augmented reality system to aid surgeons during knots tying for sutures. The forces are displayed graphically as force levels on top of the tool to substitute the haptic feedback. Although instruments are tracked using vision-based methods, these methods use force sensors or control modules to estimate force magnitude.

In contrast to the previous approaches, our method relies on a fully vision-based pipeline for estimating force feedback during RMIS. Our contributions are twofold:

- We first propose a method to translate, without preoperative data, stereo-endoscopic images to 3D biomechanical maps

- Second, we formulate instrument/tissue contact as a constraint minimization problem resulting in a linear system that can be solved in real-time, making the whole pipeline adequate for surgical tasks

To the best of our knowledge, no similar method has yet been proposed.

\section{Method Overview}

Our system aims at estimating the force around the surgical tool when in contact with a living tissue, using only the endoscopic image as input as shown in the pipeline of Figure 2. Our system consists of two stages: building and computing.

The building stage allows to obtain, from a pair of endoscopic images, the 3D geometry of the scene underlaid with a real-time biomechanical model. If we denote $\left(I_{l}, I_{r}\right)^{t}$ an image pair at a chosen time $t$, the building stage aims at obtaining the volume $\mathrm{V}$ through the building function $\Phi$ :

$$
\mathrm{V}=\Phi\left(\mathbf{C}_{l}, \mathbf{C}_{r}, I_{l}, I_{r}, n, \mathbf{b}, \gamma\right)
$$

where $\mathbf{C}_{l}$ and $\mathbf{C}_{r}$ are the camera projection matrices of left and right eye respectively and relate the 3D model in world coordinates to its $2 \mathrm{D}$ projection in pixel coordinates, $n$ the resolution of the 3D map (number of elements), $\mathbf{b}$ a set of fixed points from the 3D map and $\gamma$ represents the organ's physical properties set by the operator.

Building the volume map of the scene is done on a static phase of the surgery. The operator does not manipulate the tools and the camera is assumed to be fixed. Although the tissues are always moving due to breathing and heart motion, this motion is considered as cyclic and the map geometry remains valid.

On the other hand, the computing stage is done dynamically over time. The surgical tool is tracked frame-by-frame in 3D, and its contact with the tissue is detected. This contact is modelled as boundary condition acting on the surface of the biomechanical model represented by the volume $\mathrm{V}$. The model is constrained in position and responds by generating forces following its material law and properties $\gamma$.

These forces are assembled around the instrument tip to estimate the haptic feedback magnitude $F_{f}$ while its perception is projected back to the endoscopic image composing the image $I_{f}$ that should be visualized by the surgeon.

Our method involves determining $F_{f}$ and $I_{f}$ efficiently, in real-time and without cumbersome input from the surgeon. Returning the force to the master-side console interface is not addressed in our work and is considered to be beyond the current method.

\section{3D Physical Map of the Scene}

Processing laparoscopic images to build a biomechanical 3D map $V$ through the function $\Phi$ is a crucial step in our method. Indeed, since contact force is estimated as an instrument/tissue proximity test, obtaining a correct geometry of the scene directly impacts the force computation. Therefore, our goal is to obtain a smooth 3D shape of the organ from 2D laparoscopic images $\left(I_{l}, I_{r}\right)^{t}$. To do so, we first estimate an organ shape, that we denote $\mathrm{S}=\left\{s_{i} \in \mathbb{R}^{3}\right\}$, using well-established stereoscopy techniques [15]. More precisely, we rely on a growing scheme method proposed in [16] that permits to obtain a quasi-dense depth map. After obtaining the depth map, a triangulation is 
performed using camera matrices $\mathbf{C}_{l}, \mathbf{C}_{r}$ estimated beforehand, to finally generate the $3 \mathrm{D}$ point cloud $\mathrm{S}$.

This 3D point cloud is scattered, noisy and may contain outliers due to uncertainty coming from calibration. We thus estimate a new, smoothed 3D surface, denoted $\mathrm{S}^{*}$ by filtering out the noisy elements of S. For this purpose, we use the Cocone surface reconstruction algorithm [17] that, in contrast to widely used smoothing algorithms [18], preserves the initial geometry, especially curvatures and normals.

Although the scene geometry $\mathrm{S}^{*}$ permits to detect the contact between the instrument and the tissue, it does not allow to compute any force, thus, an underlying physical model is needed. This physical model is built using Finite Element Method to discretize the geometry and obtain a volume.

Obtaining the volume $V$ from the surface $S^{*}$ is straightforward and follows a 3D Delaunay voxelization scheme. This algorithm is very convenient, since it builds the volume on-thefly while efficiently dealing with holes and non-convex areas that may be present in $S^{*}$. We choose tetrahedral elements to represent the volume. The number of elements $n$ is to be chosen adequately in order to ensure interactive performance as well as sufficient accuracy.

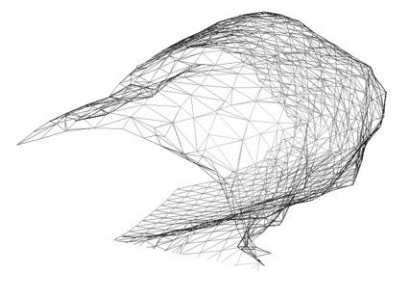

(a)

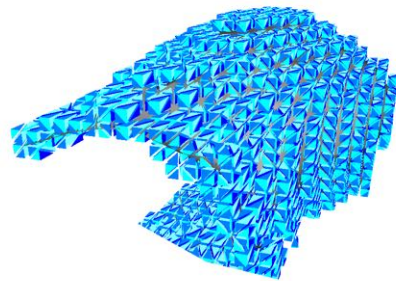

(b)
Fig. 3: 3D biomechanical map building: from the 3D shape of the organ (here the liver) obtained from stereo reconstruction, a volume is built on-the-fly to support the biomechanical model (b).

Tracking the tip of the instrument in 3D follows a similar process, however without the need of volume representation since it is a rigid body. The instrument is detected using image segmentation on left and right images and tracked over frames using classical optical flow techniques [19]. Its 3D position is recovered using triangulation and is stored in the vector $\mathbf{p}$.

\section{INSTRUMENT/Tissue CONTACT Estimation}

Among state-of-the-art elastic models [20], we choose the Saint Venant-Kirchhoff model [21] [1] for modeling organ deformations. It handles non-linear deformations and shows rotational invariance while allowing for real time computations. In addition, it can be parameterized with very few parameters $\gamma$.

The deformation is specified by the displacements of nodal positions $\mathbf{u}$ of the volume $\mathrm{V}$ and the nodal forces $\mathbf{f}$. The equation of a deformation will therefore take the form:

$$
\mathbf{K u}=\mathbf{f}
$$

The matrix $\mathbf{K}$ is the stiffness matrix, and can be computed thanks to the elastic parameters of the material $E$ and $v$. $E$ is Young's modulus and is a measure of the stiffness of the material while $v$ is Poisson's ratio and estimates the compressibility of the material.

We aim at recovering the external force $\mathbf{f}$ from the displacement of the tip of the instrument. To do so, we propose to consider the $3 \mathrm{D}$ positions of the instrument as constraints that will impose the motion of the mechanical model. The displacement of the instrument will generate forces according to the stress/strain relation of equation (2).

Assuming the 3D displacement of the instrument tip is stored in the vector $\mathbf{p}$, these constraints are modeled by adjoined $2 m$ Lagrangian multipliers collected in vector $\lambda$ that yields the multiplier-augmented form

$$
\left[\begin{array}{cc}
\mathbf{K} & \mathbf{J}^{\top} \\
\mathbf{J} & \mathbf{0}
\end{array}\right]\left[\begin{array}{l}
\mathbf{u} \\
\lambda
\end{array}\right]=\left[\begin{array}{l}
\mathbf{f} \\
\mathbf{p}
\end{array}\right]
$$

The stiffness matrix $\mathbf{K}$ is bordered with $\mathbf{J}$ and $\mathbf{J}^{\top}$, where the vector $\mathbf{p}$ contains the instrument displacement extracted from the image, while the vector $\lambda$ can be interpreted as forces required to maintain the equilibrium between internal and external forces.

The matrix $\mathbf{J}$ permits to compose a unique linear system considering the instrument as a boundary condition to the system, however this is only valid once a contact occurs. Thus, the sparse matrix $\mathbf{J}$ of size $2 m \times 3 n$ is built so that

$$
\mathbf{J}_{n}= \begin{cases}\mathbf{I}, & \text { if }\left\|p_{i}(t)-u_{i}\right\| \leq \mathscr{T} \\ \mathbf{0}_{(2,3)}, & \text { otherwise }\end{cases}
$$

where the condition $\left\|p_{i}(t)-u_{i}(t)\right\| \leq \mathscr{T}$ is a proximity distance test between the 3D positions of the instrument tip $p_{i}$ and the organ $u_{i}$, with $\mathscr{T}$ being a pre-defined threshold set empirically. The pair $\left(p_{i}, u_{i}\right)$ is composed through a radius nearest neighbor search. In other words, when $p_{i}$ is paired with $u_{i}$ a link is built following the condition 4 leading $u_{i}$ to follow the displacement of $p_{i}$.

A mean value is calculated from the forces $\mathbf{f}$ around the elements that are in contact with the instrument to finally obtain the magnitude of the force feedback $F_{f}$.

\section{VisUAL ForCE FEEDBACK}

Visual force feedback is considered as important as the haptic force feedback to improve the surgeon's perception. We consider the von Mises stress as a reliable perception of the force $F_{f}$ around the contact location. This stress can easily be computed from the stress and strain tensors that describe the material law. More precisely, the von Mises stress can be computed on each element $e$ from the normal stresses $\sigma_{x}, \sigma_{y}, \sigma_{z}$ and the shear stresses $\tau_{x y}, \tau_{y z}, \tau_{z x}$ following:

$$
\begin{aligned}
\sigma_{v m}=\frac{1}{\sqrt{2}}\left[\left(\sigma_{x}-\sigma_{y}\right)^{2}+\left(\sigma_{y}-\sigma_{z}\right)^{2}\right. & +\left(\sigma_{z}-\sigma_{x}\right)^{2} \\
& \left.+6\left(\tau_{x y}^{2}+\tau_{y z}^{2}+\tau_{z x}^{2}\right)\right]^{\frac{1}{2}}
\end{aligned}
$$

Once computed, the stress is projected back on the input left image $I_{l}$ using the camera matrix $\mathbf{C}_{l}$ and the 3D volume 
positions $\mathbf{u}$ to produce the final composition image $I_{f}$ as follows:

$$
I_{f}=I_{l} \circ\left[\mathbf{C}_{l} \cdot \Lambda\left(\mathbf{u}, F_{f}, \sigma_{v m}\right)\right]
$$

where $\Lambda(\cdot)$ is a function that generates the color map on each element $e$ using adjacent nodes $\mathbf{u}_{n_{e}}$ of the force $F_{f}$ and element's stress $\sigma_{v m}^{e}$.

\section{EXPERIMENTS AND RESULTS}

\section{A. Experiments on in-vivo Surgical Data}

Our approach was tested on a laparoscopic sequence taken during real examination on both human and porcine liver. Our purpose is to demonstrate the practical use of our method during real surgical conditions, in the presence of specular lights, beating heart and respiratory motion. The biomechanical 3D map built from stereoscopic images is composed of 1344 linear P1 tetrahedral elements for the human liver and 767 linear P1 tetrahedral elements for the porcine liver. We used elastic parameters according to [1]: Young's modulus of the liver $E_{l}=27 \mathrm{KPa}$ and Poisson's ratio $v_{l}=0.45$. Force estimation and instrument/tissue contact test was stable in realtime: refresh rate of 25 FPS was achieved with $960 \times 540$ images acquired from the Da Vinci Robot from Intuitive

The results illustrated in Figures 4 and 5 show two different visualizations of force feedback and tissue stress as well as the measured force at the tip of the instrument.
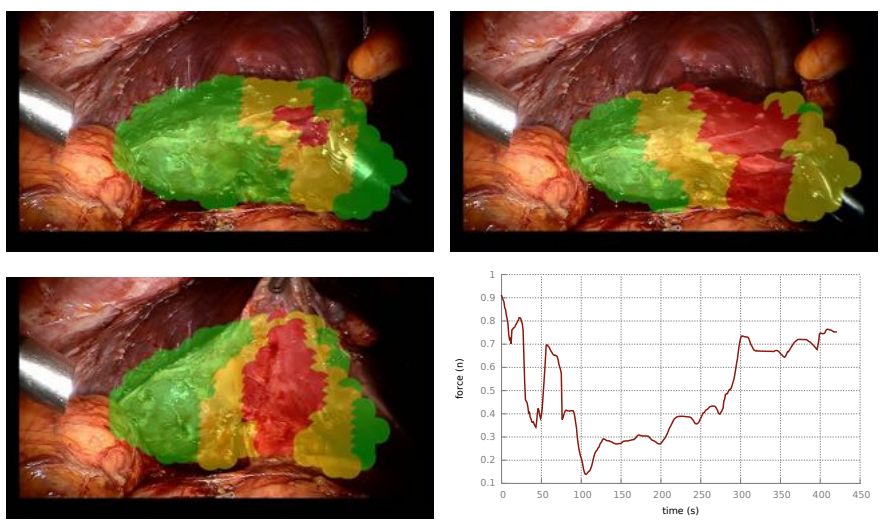

Fig. 4: Experiment on in vivo human liver: visual force feedback displayed as color map on the biomechanical 3D map showing the stress issued from instrument pulling (selected frames). The measured force at the tip of the instrument is represented in the chart.

\section{B. Experiments on DejaVu surgical data}

We evaluate our method using a DejaVu simulation scheme [22]. This simulation permits the generation of a new stereo sequence that mimics physical realism in terms of tissue response while maintaining visual fidelity from a real surgical case. This enables us to have a full ground-truth data with known tissue properties, ligament position, camera calibration parameters and the exact value of external forces (surgical instrument for instance)
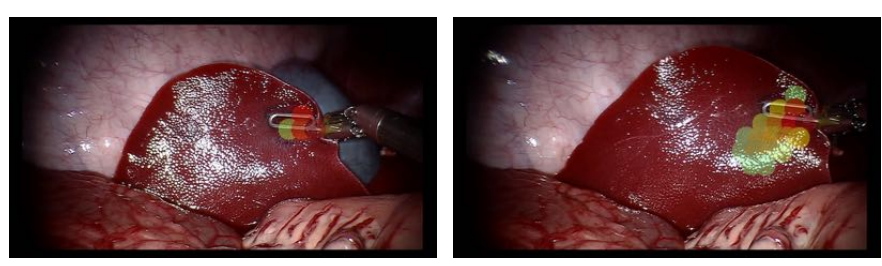

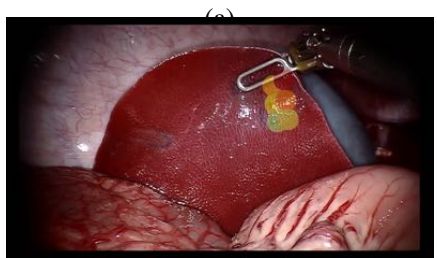

(c)

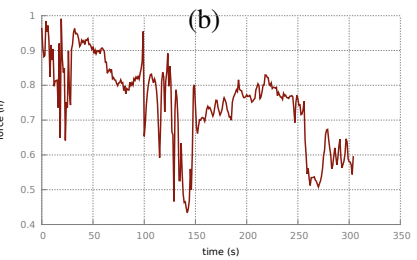

(d)
Fig. 5: Experiment on in vivo porcine liver: visual force feedback displayed around the instrument (selected frames). The measured force at the tip of the instrument is represented in the chart.

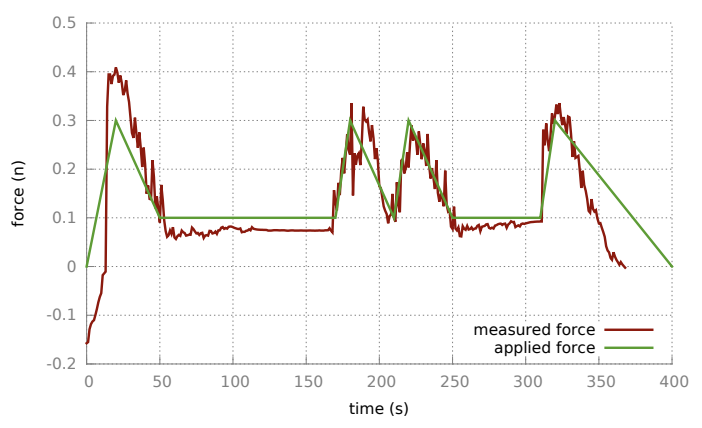

Fig. 6: DejaVu experiment: Comparison with ground-truth. Our method is able to correctly estimate the applied force at the tip of the instrument over time.

Our experiments imply a uterus surgery with the organ interacting with a surgical tool through pulling and pushing on the surface (see Fig. 2). The uterus is modeled from pre-operative data that output a volume composed of 550 tetrahedral P1 elements. It is restricted to small deformations and rotations around its attachments with the physical parameters: $E_{u}=400$ $\mathrm{kPa}, v_{u}=0.35$. The attachments $\mathbf{b}$ are pre-defined as a subset of fixed nodes from $\mathbf{u}$. A stereoscopic video is generated using a virtual camera with parameters similar to the Da Vinci robot, with a baseline $b=(5,0,0) \mathrm{mm}$ and focal $l=700$. In order to obtain a realistic rendering, illumination and texturing are considered. Light position, diffuse reflectance, specular reflection and roughness are estimated from the original image and the scene texture is mapped on the visual mesh.

a) Ground-truth comparison: Figure 6 reports the comparison between the virtually applied force and the measured force using our method over time. We can notice that our method is able to correctly estimate the applied force at the tip of the instrument over time. These results are obtained using known tissue parameters and the exact position of the attachments. However, in practice, these values are unknown 
and are most of the time set from textbooks of anatomy atlases. Thus, we propose to measure the impact of varying material properties and attachment position in measuring force feedback.

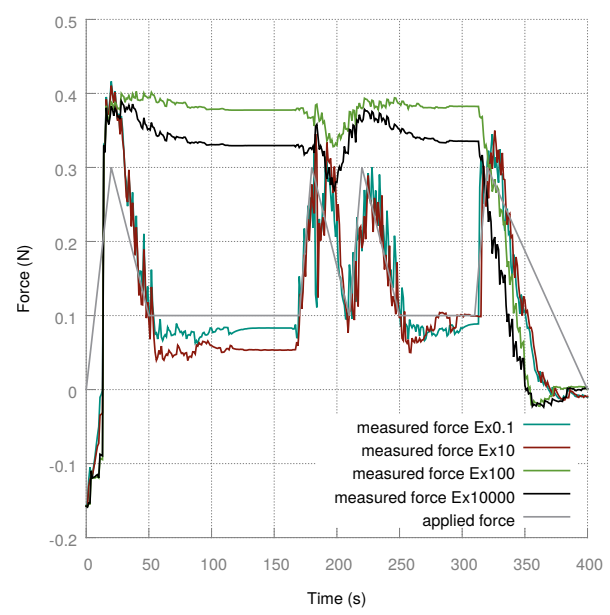

Fig. 7: DejaVu experiment: Varying material stiffness $E_{u}$.

b) Varying material parameters: We choose to vary the Young's modulus of our tissue and estimate external forces by varying $E_{u}$ in the range $E_{u} \times 10^{1}$ to $E_{u} \times 10^{4}$ while keeping the position of the attachments. The results of these variations are reported in Figure 7 and show that a slight variation of the value of $E_{u}$ does not influence the value of $F_{f}$. However, when making the tissue stiffer $\left(E_{u} \times 10^{3}\right.$ and $\left.E_{u} \times 10^{5}\right)$ the measured force differs from the gourd-truth.

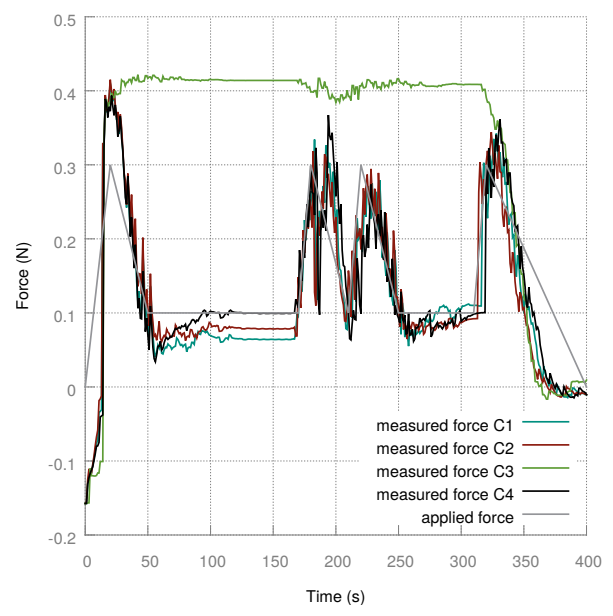

Fig. 8: DejaVu experiment: Impact of the attachment positions (4 configurations).

c) Impact of the attachment positions: We also estimate the impact of the attachments on measuring the force. These attachments represent ligaments surrounding the organ and are set manually. We tested 4 configurations with different locations and number of fixed nodes $\mathbf{b}$. The results are reported in Figure 8 and show that the location and number of fixed nodes for configurations 1,2 and 4 do not significantly impact the value of $F_{f}$. However, in the configuration 3 where fixed constraints were placed around the contact area, our method was not able to correctly estimate the force, since the stress is null at fixed nodes.

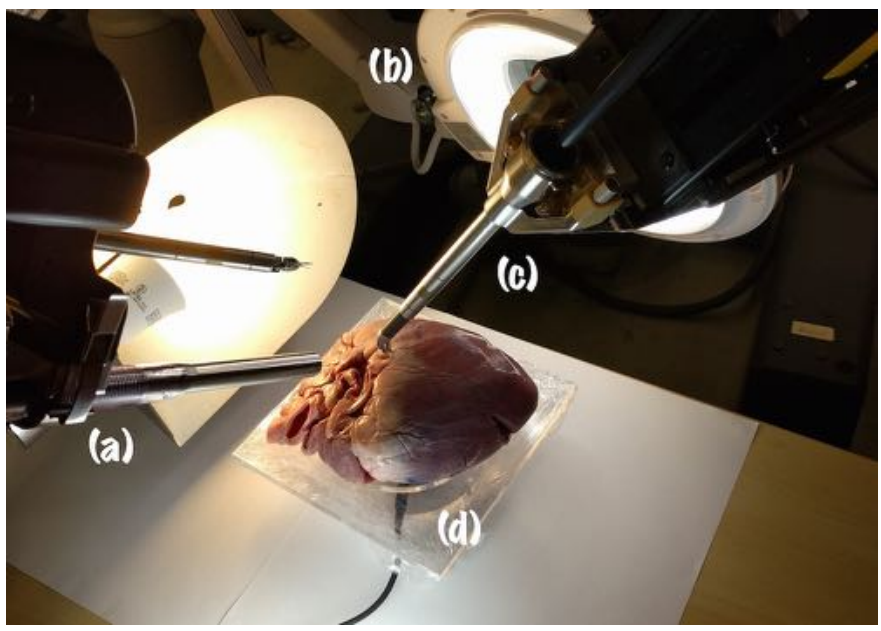

Fig. 9: Setup of the ex vivo experiment: the setup includes a stereoscopic camera (a), a light source (b), surgical tools (c) and a force sensor plate (d).

\section{Experiment on ex vivo Surgical Data}

Experiment on an ex vivo porcine heart was conducted following the setup in Figure 9. A force sensor plate (ATI Mini40 F/T Sensor) is used to measure the acting forces on the heart while being filmed by a stereoscopic camera. The fixed nodes $\mathbf{b}$ are supposed known (set underneath of the heart), however material properties are unknown and are set according to a textbook.

The chart in figure 10 reports the comparison between the ground-truth measurement and the estimated force using our method. It shows that, although the force magnitude does not exactly fit the ground-truth, its relative value and evolution over frames correspond to the ground-truth. This point is discussed in the next section.
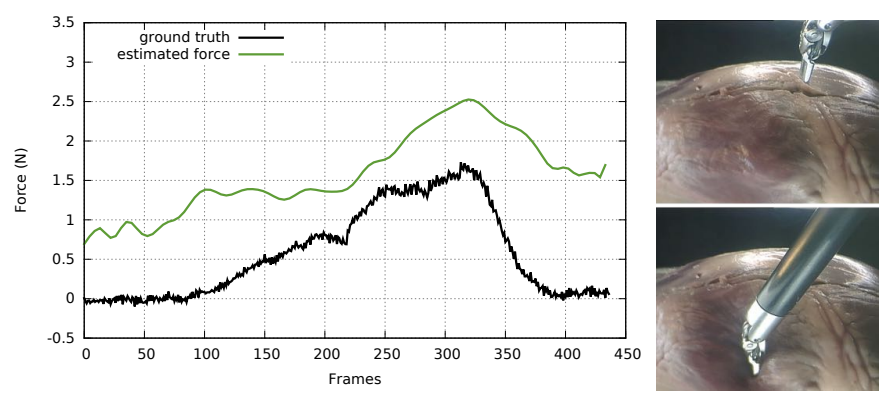

Fig. 10: ex vivo experiment: Comparison with ground-truth. The right images correspond to rest (top) and deformed (bottom) states of the heart.

\section{DISCUSSION}

The results presented here show that it is possible to estimate force feedback using solely endoscopic images. Although 
our method comes with real advantages, it has some limitations which we discuss below.

Tissue properties: the conducted experiments have demonstrated that estimating force using biomechanical models highly depends on tissue properties. This is an expected result. Fundamentally, solving this problem is challenging knowing the linear force/displacement relation of Eq. 2. However. the more pressing question is whether an absolute or relative estimation is the most valuable to surgeons [5],[4].

Structure identification: various tissues and organs usually compose a surgical scene, segmenting and labeling each component to assign different tissue properties is not supported in the current method. While creating a heterogeneous biomechanical 3D map is straightforward, identifying organs and surrounding tissue in endoscopic images is challenging. One can rely on learning approaches or purely geometric approaches [23] to classify the scene and obtain a complete $3 \mathrm{D}$ abstraction of the image.

Surgical scene motion: the surgical scene is translated from images to 3D map on-the-fly assuming a static scene. In practice, tissues are moving, and non-rigid tracking is needed to synchronize with current events. Moreover, stereo reconstruction may result in noisy surfaces due to lack of salient features and errors in triangulation which will cause the voxelization to fail. This is even more important since the instrument/tissue contact force is computed from a geometrical proximity test. Another source of failure may emanate form the optical flow that can lead to drifting. More robust methods such as the ones using learning models [24] are more adequate and can easily be plugged in our framework.

\section{CONCLUSION}

We present a vision-based approach for estimating visual and haptic force feedback for robot-assisted minimally invasive surgery. Our method takes full advantage of stereoendoscopic camera to detect instrument/tissue contact and estimate contact force. Our experiments show that our estimate can be compared with ground-truth and visual feedback can be composed over the original image.

More validation is obviously needed to asses the results. A user study with experienced and unexperienced surgeons will also permit to identify the real need in terms of visualization and perception on force feedback.

\section{REFERENCES}

[1] N. Haouchine, J. Dequidt, I. Peterlik, E. Kerrien, M.-O. Berger, and S. Cotin, "Image-guided simulation of heterogeneous tissue deformation for augmented reality during hepatic surgery." in ISMAR 2013, 2013, pp. 199-208.

[2] S. Suwelack et al., "Physics-based shape matching for intraoperative image guidance," Medical physics, vol. 41, no. 11, p. 111901, 2014.

[3] R. Plantefève, I. Peterlik, N. Haouchine, and S. Cotin, "Patient-specific biomechanical modeling for guidance during minimally-invasive hepatic surgery," Annals of Biomedical Engineering, vol. 44, no. 1, pp. 139-153, 2016. [Online]. Available: http://dx.doi.org/10.1007/s10439-015-1419-z

[4] O. AM, "Haptic feedback in robot-assisted minimally invasive surgery," Current opinion in urology, vol. 19, no. 1, pp. 102-107, 2009.

[5] E. R. Carol, A. Takintope, B. Darius, C. C. David, M. O. Allison, and D. Y. David, "Effects of visual force feedback on robot-assisted surgical task performance," The Journal of Thoracic and Cardiovascular Surgery, vol. 135, no. 1, pp. 196-202, 2007.
[6] C. J. Payne and G.-Z. Yang, "Hand-held medical robots," Annals of biomedical engineering, vol. 42, no. 8, pp. 1594-1605, 2014.

[7] A. M. Okamura, "Methods for haptic feedback in teleoperated robotassisted surgery," Industrial Robot: An International Journal, vol. 31, no. 6, pp. 499-508, 2004

[8] P. J. Berkelman, L. L. Whitcomb, R. H. Taylor, and P. Jensen, "A miniature microsurgical instrument tip force sensor for enhanced force feedback during robot-assisted manipulation," IEEE Transactions on Robotics and Automation, vol. 19, no. 5, pp. 917-921, 2003.

[9] A. Biason, G. Boschetti, A. Gasparetto, M. Giovagnoni, and V. Zanotto, "A force-torque sensor for the applications in medical robotics," in AMST05 Advanced Manufacturing Systems and Technology. Springer, 2005 , pp. 543-550.

[10] O. Mohareri, C. Schneider, and S. Salcudean, "Bimanual telerobotic surgery with asymmetric force feedback: a davinci $\AA$ surgical system implementation," in Intelligent Robots and Systems (IROS 2014), 2014 IEEE/RSJ International Conference on. IEEE, 2014, pp. 4272-4277.

[11] M. Lerotic, A. J. Chung, G. Mylonas, and G.-Z. Yang, "Pq-space based non-photorealistic rendering for augmented reality," in International Conference on Medical Image Computing and Computer-Assisted Intervention. Springer, 2007, pp. 102-109.

[12] S. Chan, P. Li, G. Locketz, K. Salisbury, and N. H. Blevins, "Highfidelity haptic and visual rendering for patient-specific simulation of temporal bone surgery," Computer Assisted Surgery, vol. 21, no. 1, pp. 85-101, 2016.

[13] M. Mura, Y. Abu-Kheil, G. Ciuti, M. Visentini-Scarzanella, A. Menciassi, P. Dario, J. Dias, and L. Seneviratne, "Vision-based haptic feedback for capsule endoscopy navigation: a proof of concept," Journal of MicroBio Robotics, vol. 11, no. 1-4, pp. 35-45, 2016.

[14] T. Akinbiyi, C. E. Reiley, S. Saha, D. Burschka, C. J. Hasser, D. D. Yuh, and A. M. Okamura, "Dynamic augmented reality for sensory substitution in robot-assisted surgical systems," in Engineering in Medicine and Biology Society, 2006. EMBS'06. 28th Annual International Conference of the IEEE. IEEE, 2006, pp. 567-570.

[15] L. Maier-Hein, P. Mountney, A. Bartoli, H. Elhawary, D. Elson, A. Groch, A. Kolb, M. Rodrigues, J. Sorger, S. Speidel, and D. Stoyanov, "Optical techniques for $3 \mathrm{~d}$ surface reconstruction in computer-assisted laparoscopic surgery," Medical Image Analysis, vol. 17, pp. 974-996, 2013.

[16] D. Stoyanov, "Stereoscopic scene flow for robotic assisted minimally invasive surgery," in International Conference on Medical Image Computing and Computer-Assisted Intervention, 2012, pp. 479-486.

[17] T. K. Dey and J. Giesen, "Discrete and computational geometry: The goodman-pollack festschrift," B. Aronov, S. Basu, J. Pach, and M. Sharir, Eds. Springer Berlin Heidelberg, 2003, ch. Detecting Undersampling in Surface Reconstruction, pp. 329-345.

[18] D. Levin, "The approximation power of moving least-squares," Math. Comput., vol. 67, no. 224, pp. 1517-1531, Oct. 1998. [Online]. Available: http://dx.doi.org/10.1090/S0025-5718-98-00974-0

[19] J.-Y. Bouguet, "Pyramidal implementation of the affine lucas kanade feature tracker description of the algorithm," Intel Corporation, vol. 5, no. 1-10, p. 4, 2001.

[20] A. Nealen, M. Müller, R. Keiser, E. Boxerman, and M. Carlson, "Physically based deformable models in computer graphics," in Computer Graphics Forum, vol. 25 (4), 2006, pp. 809-836.

[21] R. Kikuuwe, H. Tabuchi, and M. Yamamoto, "An edge-based computationally efficient formulation of saint venant-kirchhoff tetrahedral finite elements," ACM Transactions on Graphics (TOG), vol. 28, no. 1, p. 8, 2009.

[22] N. Haouchine, D. Stoyanov, F. Roy, and S. Cotin, "Dejavu: Intraoperative simulation for surgical gesture rehearsal," in Medical Image Computing and Computer Assisted Intervention - MICCAI, 2017, pp. $523-531$.

[23] N. Haouchine and S. Cotin, "Segmentation and labelling of intraoperative laparoscopic images using structure from point cloud," in IEEE International Symposium on Biomedical Imaging, ISBI, 2016, pp. 115118. [Online]. Available: https://doi.org/10.1109/ISBI.2016.7493224

[24] M. Allan, S. Thompson, M. J. Clarkson, S. Ourselin, D. J. Hawkes, J. Kelly, and D. Stoyanov, "2d-3d pose tracking of rigid instruments in minimally invasive surgery," in International Conference on Information Processing in Computer-assisted Interventions. Springer, 2014, pp. 110 . 